Alle Bestimmungen wurden als halbquantitative Analysen auf papierchromatographischem Wege an Extrakten aus gefriergetrocknetem Algenmaterial durchgeführt. Bei den untersuchten Arten konnten bis zu 18 Aminosäuren, 10 reduzierende und 2 nichtreduzierende Zucker nachgewiesen und identifiziert werden. Die Analysen stimmen mit den Ergebnissen anderer Autoren ${ }^{2}$ ), ${ }^{3}$ ) gut überein.

In Fig. 1 sind die Ergebnisse der Aminosäurebestimmungen zusammengestellt. Für Methionin, Valin, Alanin und Serin ergaben vor allem bei jüngeren Kulturen (Ernte I) dic Aneurinserien höhere Aminosäurewerte als die Kontrollen,

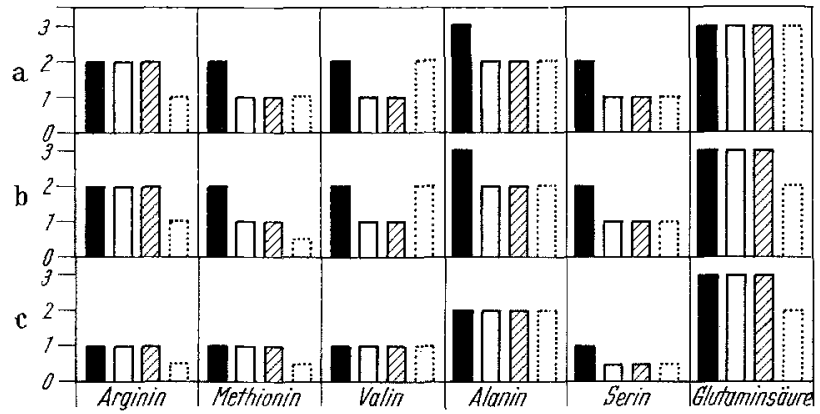

Fig. 1. Aminosäurebildung bei Chlorella pyrenoidosa unter dem Einfluß von zusätzlichem Aneurin ( $1 \mathrm{mg} / 100 \mathrm{ml}$ Nährlösung). Nährlösungskonzentration: $a \quad 3 / 2, b \quad 2 / 2 ; c c 1 / 2$. Schwarz bzw. schraffiert: Aneurinkulturen; wei : Kontrollen; schwarz und wei $\beta$ ausgezogen: frühe Ernte $(I)$; schraffiert und weiß strichliert: späte Ernte (II)

bei Glutaminsäure und Arginin traten. Unterschiede erst später, in Ernte II, auf. Histidin und Asparaginsäure ließen keine Differenzen erkennen. In älteren Kulturen, deren Zellen ihr Teilungswachstum bereits eingestellt hatten und zur Reservestoffspeicherung übergegangen waren, glichen sich die Unterschiede vielfach wieder aus, indem die Werte der Aneurinserien auf die meist unveränderten Kontrollwerte absanken (Fig. 1, Ernte II).

Die meisten Versuche wurden mit Chorela pyrewoidosa durchgeführt. Orientierende Untersuchungen mit anderen.

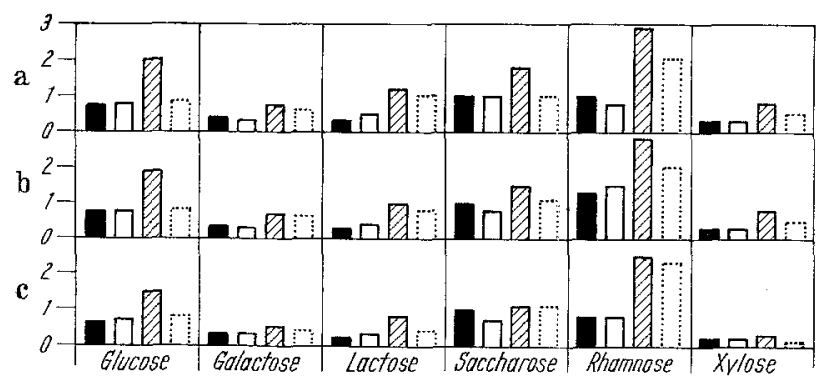

Fig. 2. Zuckergehalt bei Chlorella pyrenoidosa unter dem Einfluß von zusätzlichem Aneurin (Signaturen und Bezeichnung wie bei Fig. 1)

Algen brachten bei Stichococcus bacillaris und Hormidium flaccidum gleichsinnige Ergebnisse, in geringerem Maße auch bei Scenedesmus acuminatus und Ankistrodesmus atfinis.

Noch stärker als bei den Aminosäuren waren die Ausschläge bei den Zuckern (Fig. 2), wobei besonders auffällt, daß diese im Gegensatz zu den Aminosäuren ganz allgemein in den älteven Kulturen in größeren Mengen gefunden wurden. Zum Teil (Glucose, Lactose) war die fördernde Wirkung des Aminosäurezusatzes überhaupt erst bei der zweiten Ernte erkennbar. Im gleichen Sinne wie die in Fig. 2 angeführten Zucker reagierte auch Ribose; weitere $Z$ ucker konnten meist nur in ganz geringen Mengen nachgewiesen werden und blieben unberìcksichtigt. Auch hier wutden stichproben. weise andere Algen in die Untersuchung mit einbezogen. Positive Ergebnisse erbrachten die Analysen bei Ankistrodesmus affinis und Hormidium flaccidum, während Stichococcus bacillaris bei an sich sehr geringem Zuckergehalt im Durchschnitt keine Aneurinwirkung erkennen ließ.

Eine ausführlichere Darstellung und Diskussion dieser und weiterer, hier nicht berührter Ergebnisse an Kulturen in Nähr- und Mangellösungen verschiedener Zusammensetzung wird durch den einen von uns (W. ScHÜKERK) zu einem späteren Zeitpunkt erfolgen.
Der deutschen Forschungsgemeinschaft danken wir für Förderung durch eine Sachbeihilfe.

Botanisches Institut der Technischen Hochschule München in Weihenstephan

H. v. WITSCH und W. SchüKERK

Eingegangen am 22. Oktober 1958

1) Witsch, H.v.: Die Algenkultur. In W. Ruhlax̃d Handbuch der Pflanzenphysiologie, Bd.IV, S. 90-99. 1958.-2) BuRLEw, J. S.: Carnegie Inst. Wash. Publ. 1953, 600. -3) Morimura, Y. it. N. TAmiYA: Food Technol. 8, 179 (1954).

\section{Atmungsreaktionen in Urethan-Narkose auf exterozeptive Reize und deren Beeinflussung durch Pharmaka}

An der weißen Ratte in Urethan-Narkose konnte nach mechanischen, osmotischen, thermischen, chemischen und elektrischen Reizen von Exterozeptoren eine Verstärkung der Atmung und eine Blutdruckänderung registriert werden. Am besten bewährte sich eine elektrische Reizung wie bei der Analgesie-Messung. Während dieses Reizes waren Atemvolumen, -amplitude und -frequenz vergrößert. Mit dieser Methode ließen sich über mehrere Stunden reproduzierbare Werte aufzeichnen. Änderungen der Atmung und des Blutdruckes durch Reizung von Exterozeptoren sind bekannt, unseres Wissens wurde jedoch in Narkose eine pharmakologische Beeinflussung bisher nicht beschrieben.

Die entsprechend der Reizstärke maximale Reizatmung wurde durch Deumacard (Pentamethylentetrazol) oder Eukraton (Methyl-äthyl-glatarsäureimid) nicht verstärkt, dagegen die Reizatmung bei untermaximaler Reizung, wobei die Spontanatmung nicht nennenswert vergrößert wurde. $1 \mathrm{mg} / \mathrm{kg}$ Opilon (6-Azetoxy-thymoxyäthyldimethylaminohydrochlorid) intravenös, $10 \mathrm{mg} / \mathrm{kg}$ Regitin (2 [N-p-Tolyl-N-m-oxyphenylaminomethyl]-imidazolin. methansulfonic.) intravenös und 1 bis $5 \mathrm{mg}$ pro $\mathrm{kg}$ Morphin intravenös verminderten die Reizatmung oder hoben sie auf, Deumacard und Eukraton wirkten antagonistisch. 0,5 bis $1 \mathrm{mg} / \mathrm{kg}$ Chlorpromazin intravenös blockierte die Reizatmung, Eukraton und Pentedrin waren danach wirkungslos. $5 \mathrm{mg} / \mathrm{kg}$ Pendiomid und $9 \mathrm{mg} / \mathrm{kg}$ Hexamethonium zeigten keine Abschwächung der Reizatmung. Bei der Abkühlung der Tiere verminderte sich die Reizatmung deutlich, die kritische Temperatur lag zwischen 29 und $31^{\circ} \mathrm{C}$.

Eine ausführliche Veröffentlichung wird an anderer Stelle erfolgen.

Dresden A 1, Pharmakologisches Institut der Medizinischen Akademie ,Carl Gustav Carus" (Direktor: Prof. Dr. W.OErssNER)

Eingegangen am 31. Oktober 1958

KaRI FELLER

\section{Die Wirkung des Pantethins auf das Coenzym A \\ der Leber und Niere bei experimenteller Nephrose}

Die beim Versuchstier durch Antinierenserum hervorgerufene Hyperlipämie ist begleitet von einem Absinken des Gehalts an Coenzym A in Leber und Niere, welche durch Behandlung mit Adenosintriphosphat teilweise rückgängig gemacht werden kann ${ }^{1}$; diese Tatsache wird gedeutet als eine Folge der verbesserten Phosphorylationsprozesse welche zur Synthese des Coenzyms A erforderlich, jedoch bei der Nephrose verändert sind.

Für die ersten Stufen der Biosynthese dieses wichtigen Enzyms ist es außer angemessenen Konzentrationen des Adenosintriphosphats unerläBlich, daß Cystein und selbstverständlich auch Pantothensäure in normalen Mengen zur Verfügung stehen.

Es konnte nämlich nachgewiesen werden, daß die Synthese des Coenzyms A in folgender Weise verläuft ${ }^{2}$ ):

$$
\begin{gathered}
\text { Pantothensäure }+ \text { Cystein } \stackrel{\text { ATP }}{\longrightarrow} \text { Pantothenilcystein } \stackrel{-\mathrm{CO}_{3}}{\longrightarrow} \\
\text { Pantethein } \stackrel{\text { ATP }}{\longrightarrow} \text { Pantethein-4'-Phosphat } \stackrel{\text { ATP }}{\longrightarrow} \\
\text { Dephospho-COA } \stackrel{\text { ATP }}{\longrightarrow} \text { CoA. }
\end{gathered}
$$

Auf diesen Voraussetzungen fußend, wurde die nachfolgende Untersuchung mit dem Ziel ausgeführt, die Wirkung des Pantethins auf das Coenzym A in der Leber und Niere von mit nephrotoxischem Serum behandelten Tieren festzustellen, einer Substanz, die aus 2 Molekeln Pantethin besteht, welche durch eine leicht reduzierbare und infolgedessen im tierischen Organismus leicht spaltbare -S-S-Brücke miteinander ver- 